\title{
Überlegungen zu der sumerischen Zeichen-Gruppe ŠÈ.KA*
}

\author{
Marcos SUCH-GUTIÉRREZ \\ Instituo de Filología - CSIC, Madrid
}

In den Texten aus dem 3. Jt. beschränkt sich die Zeichen-Gruppe ŠÈ.KA mit Ausnahme eines altakkadischen Briefes aus Adab (s. unten Abschnitt 2) auf die Ur-III Zeit ${ }^{1}$. In dieser Periode findet sich

* msuch@filol.csic.es.

Mein besonderer Dank gilt M. Molina für die Erlaubnis, Bücher aus seiner privaten Bibliothek zu verwenden u. für die Durchsicht des Manuskriptes. Für die Durchsicht des Manuskriptes möchte ich auch Cl. Fischer sehr herzlich danken.

Die Abkürzungen richten sich nach R. BORGER, Handbuch der Keilschriftliteratur 1-2 (Berlin - New York 1967-1975), M. SigRIST u. T. GoMI, The Comprehensive Catalogue of Published Ur III Tablets (Bethesda, Maryland 1991), u. W. SAllaberger u. A. WeStenHOLZ, Akkade-Zeit und Ur III-Zeit, OBO 160/3 (Freiburg - Göttingen 1999) S. 393 ff. Ferner sind noch folgende Abkürzungen zu berücksichtigen: Buffalo SNS 11/2= M. I. HUSSEY, «Babylonian Tablets in the Museum of the Buffalo Society of Natural Sciences», Bulletin of the Buffalo Society of Natural Sciences 11/2 (1915) S. 109-160; MAEKAwA, Priests = K. MAEKAWA, «The 'Temples' and the 'Temple Personnel' of Ur III Girsu-Lagash», in Priests and Officials in the Ancient Near East, Hrg. K. WATANABE (Heidelberg 1999) S. 61-102; Michail = G. PETTINATO, L'uomo cominciò a scrivere. Iscrizioni cuneiformi della Collezione Michail (Milano 1997); MichaLOWSKI, LEM = P. MiCHALOWsKI, Letters from Early Mesopotamia (Atlanta, Georgia 1993); PETtinato, $S V S 1 / 3=$ G. PetTinato, Studi per il vocabolario sumerico 1/3. G. Reisner, Tempelurkunden aus Telloh. Glossario (Roma 1985).

1 Hier bleiben Fälle, in denen KA grammatikalische Elemente darstellt, außer Betracht, z.B. má-ŠÈ.KA in dem Umma-Text MVN 14 69: 5 (ك̌ 45/AS 2 / -) ist als má-zì(-da)-ka -(Auslaut+a)k+Lokativ (-a)- zu interpretieren, vgl. z.B. die Umma-Texte TÉNS 217: 4 (Š 48 / xi -), SACT 2 182: 3 (AS 5 / viii -), UTI 4 2466: 3 (AS 9 / iii -), TPTS 217: 3 (ŠS 1 / -) u. UCP 9/2/2: 4-5 (ŠS 4 / -). Bei má-Š̀̀.KA-Šè in dem Lagaš-Text SAT $1374: 2$ (AS $5 /$ iv -) ist aufgrund der

Sefarad 63 (2003) págs. 393-410

(C) CSIC

ISSN 037-0894 
ŠÈ.KA fast nur in der Provinz Lagaš. Außerhalb von Lagaš ist mir ŠÈ.KA mit Lesung éš-kìri, 'Nasenseil', nur im Text TCNY 393 Rs. 8 (Š 42 / -) aus Umma bekannt:

$$
\begin{array}{ll}
2 \text { ma-na síg ùz // } & \text { «2 Minen (c. } 1 \text { kg) Ziegenwolle } \\
\text { éš-kìri sá-úr sá-pa-š[è], } & \begin{array}{l}
\text { für die Nasenseile (der) sá-úr- (und) sá-pa } \\
\text { (Teile des Geschirrs)» }{ }^{2} .
\end{array}
\end{array}
$$

In Lagaš kommt ŠÈ.KA meines Wissens in drei Kontexten vor:

1) Im Ausdruck PN-ra éš(ŠÈ) kìri(KA)(-šè)...dù/ur ${ }_{5}$, «jemandem ein Seil an der Nase anmachen» ${ }^{3}$.

2) In Zusammenhang mit der Abgabe von Naturalien, vor allem von Gerste und Mehl ${ }^{4}$.

3) In den sogenannten «Botentexten», die Bier, Brot, Öl (und Schaffett) als Versorgung für eine Reise in Zusammenhang mit ŠÈ.KA erwähnen ${ }^{5}$.

beiden anderen Lagaš-Texte Nik 2 177: 3 (AS 5 / -) u. ITT 5 6986: 2 (AS 8 / -) als má-zì(-da)-ka-šè -(Auslaut+a)k+a(k)+Terminativ (-šè)- zu interpretieren.

2 Zur Passage s. A. SALONEn, Hippol., S. 127, dasselbe in IDEM, Agricultura, S. 105. Zu sá-úr u. sá-pa als Teile des Geschirrs s. J. BAUER, Altsumerische Wirtschaftstexte aus Lagasch, Studia Pohl 9 (Roma 1972) S. 638.

3 Siehe dazu zuletzt M. Molina u. M. Such-GutiérRez, «On Terms for Cutting Plants and Noses in Ancient Sumer», JNES 63/1 (2004) (im Druck). Ein weiterer Beleg ist RA 84 S. 169 3: 3 (-/-). Hierher gehört wohl der Ausdruck éren éš? (ŠÈ) kìri?(KA) (ur 5 -ra), "Arbeitstruppen, (denen) ein Seil? an der Nase? (angemacht worden ist)», ASJ 20 S. 99 f. Text 2: III 2', Rs. VII 5 (ك̌ $46 /$-), MVN 2 72: 2 (Š $46 /$ x -) -auf der Hülle auch in Vs. 7-, u. á (éren) éš? (ŠÈ) kìri?(KA) nu-ur ${ }_{5}$-ra, «Lohn (der) Arbeitstruppen, (denen) ein Seil? an der Nase? nicht angemacht worden ist», MVN 911 Rs. 18 ( $\breve{S} 47$ / xi -). Zur Bestimmung beider Ausdrücke würde die Deutung von éren-sag-AŠ weiterhelfen, die in den

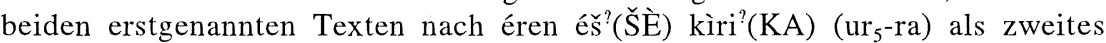
Glied von éren-bala-tuš-a vorkommt. Trotzdem entzieht sich die Deutung des Terminus meiner Kenntnisse. Zu éren-bala-tuš-a s. K. MAEKAwA, «The erínPeople in Lagash of Ur III Times», RA 70 (1976) S. 19 ff.

${ }^{4}$ Zur Aufzählung der Belege s. Appendix. Bei ITT 56939 Rs. 3' ([?] / [?]) ist der Text derart abgebrochen, so daß der Zusammenhang von ŠÈ.KA kaum zu bestimmen ist.

5 TCTI 2 3791: 1-4 (- / ix -), DAS 137 Rs. 13-15 (- / $\left.\mathrm{xi}^{2}-\right)$ u. DAS 150: 8-10 $\left(-/ \mathrm{xi}^{2}-\right)$. 
ŠÈ.KA hat man in den zwei letztgenannten Kontexten generell als zíd/zì-ka, zíd/zì-KA oder ZÍD/Zİ.KA gelesen und als 'zu Mehl verarbeiten' oder als eine Mehlsorte gedeutet ${ }^{6}$. Im vorliegenden Beitrag versuche ich aufzuzeigen, daß Š̀̇.KA in den beiden letztgenannten Kontexten eine Gabe, vor allem von Mehl, bezeichnete.

\section{LESUNG UND DEUTUNG VON ŠÈ.KA ANHAND DER UR-III-ZEITLICHEN TEXTE}

Die erste Frage, die beantwortet werden muß, ist die der Lesung der Zeichen ŠÈ.KA. Hier könnte man außer dem oben erwähnten zì$\mathrm{ka} / \mathrm{KA}$ an éš-kìri su(r) $\mathrm{ra}_{13}$ oder zír ${ }^{7}$ denken. Die in Appendix und Anm. 5 angeführten Quellen geben keinen direkten Aufschluß für eine Lesung (z.B. Auslaut des Wortes), jedoch existieren indirekte Hinweise.

Einen ersten Anhaltspunkt liefert TCTI 2 3579: 1 (ŠS 9 / ii -): Hier werden die beiden Zeichen als zwei Wörter verstanden, wobei das erste Zeichen (Š̉̇) als zì zu lesen ist, da beide Zeichen durch das Hohlmaß gur getrennt werden:

\footnotetext{
${ }^{6}$ Siehe dazu Th. G. PInches, The Amherst Tablets. I (London 1908) S. 169, S. 180 Anm. zu Text 104; F. Thureau-Dangin, Textes de l'époque d'Agadé, ITT 1 (Paris 1910) S. 50 Anm. 1; B. LANDSBERGER, «Zur Mehl Bereitung im Altertum», OLZ 25 (1922) S. 339; B. LAFONT, Documents administatifs sumériens provenant du site de Tello et conservés au Musée du Louvre (Paris 1985) S. 53 Anm. zu Text 137; IDEM, «Nouvelles lettres du temps du rois d'Ur», RA 84 (1990) S. $168 \mathrm{~N}^{\circ}$ 3: 3; P. Michalowski, LEM, S. 79 f. 129 , S. 82 138; u. K. MAEKAWA, Priests, S. 89. K MAEKAWA läßt dagegen den Ausdruck ohne Übersetzung in $R A 70$ (1976) S. 11 Demgegenüber hat A. DEIMEL, $\breve{S} L 2 / 4,53690$ d ŠÈ.KA als einen «Bürovermerk für abgeliefertes(?) Getreide» gedeutet, dazu vgl. auch IDEM, «Ur-III-Texte aus der Sammlung Wengler», OrSP 5 (1930) S. 50 Anm. zu Text 11, u. G. Pettinato, SVS $1 / 3$ hat den Terminus dagegen als «Verwaltungsterminus, der sich auf Darlehen bezieht» übersetzt.

7 Die Lautwerte su(r)ra $a_{13}$ u. zír sind mir nur aus den lexikalischen Listen bekannt, s. E. I. GoRDON, «Sumerian animal proverbs and fables: Collection five», JCS 12 (1958) S. 72 Anm. 6 u. F. EllermeIER, Sumerisches Glossar (Göttingen 1980) S. 544.
} 


\author{
4.1.0 zì gur $\mathrm{KA}$, \\ še-šuku-ra šabra, \\ má-a si-ga,
}

$$
\text { «12601 Mehl (für) KA }{ }^{8}
$$

Verpflegungsgerste (des) Šabra-Verwalter(s), die auf das Schiff geladen worden ist».

Ein zweiter Hinweis auf die Lesung zì des Zeichens ŠÈ ist, daß zì-KA ein aus Getreide verarbeitetes Produkt bezeichnet, worauf folgende Elemente deuten:

1) (še) zì-KA wird, gleich anderen mit Hohlmaß gemessenen Produkten (z.B. Brot, Getreide, Mehl oder Sesamöl), in einigen Textstellen vor gur angeführt ${ }^{9}$.

2) Außer Gerste wird auch zíz, 'Emmer', für zì-KA verwendet ${ }^{10}$.

3) Neben zì-KA erwähnen die «Botentexte» auch Reisen wegen der Verarbeitung anderer Produkte ${ }^{11}$.

4) Wahrscheinlich der Ausdruck KU zì-KA ${ }^{12}$, den ich nicht deuten kann, der mir aber in Verbindung mit Gerste und Mehl bekannt ist ${ }^{13}$.

\footnotetext{
${ }^{8}$ Da es sich um Gerste (še-šuku-ra) handelt, ist anzunehmen, daß der Schreiber eigentlich 4.1.0 še gur zì-KA meinte, vgl. z.B. ASJ 3 S. 175 175: 1 (Š 45/AS 2 / xii -), oder 4.1.0 še zì-KA gur, vgl. ebenso $M V N 6$ 328: 1 (Š 37 / -).

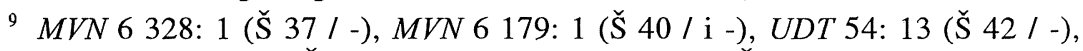
CT 519 12912: III 14 (Š 44 / -) u. $M V N 1196$ Rs. 28 (Š 44 / -).

${ }^{10}$ MVN 12 23: 1-2 (̌̌ 46/AS 3 / iv -), TCTI 2 3779: 1-2 (ŠS 9 / ii -) u. TCTI 2 4318: 2-3 (ŠS 9 / -).

${ }^{11}$ z.B. $M V N 10$ 163: 9 (- / i 22): 0.0.0.8 sìla àga-ús kaš ninda-šè'(TÚG) DU, «8 $1<>$, (wenn) der Leibwächter zum Bier und Brot geht», MVN 5253 Rs. 7-9 (- I xi 15): 0.0.0.5 sìla kaš 0.0.0.5 sìla ninda, 2 gín ì, $I$-din- ${ }^{\mathrm{d}} A d a d$ dabin-KA-šè ginna, «5 1 Bier, 51 Brot (und) 2 Schekel Öl, (wenn) Iddinadad zum KA-Gerstenmehl ging» u. $D A S$ 187: 9-12 (- / $\left.\mathrm{xi}^{2} 13\right)$ : 0.0.0.2 sìla kaš 0.0.0.2 sìla ninda, 2 gín ì, Ša-ru-um-ba-ni, dabin-KA-uru-šè gin-na, «2 1 Bier, 21 Brot, 2 Schekel Öl, (wenn) Šarrumbān̄̄ zum KA-Gerstenmehl (der) Stadt ging».

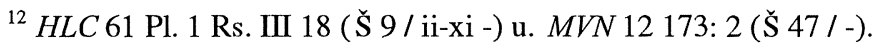

${ }^{13} \mathrm{KU}$ še: $M V N 1287$ (Š $48 / \mathrm{x}$-), BE $3^{1} 84$ Rs. IV 81 (Š 48 / viii -), MVN 417 Rs. 3 (AS 7 / iii 3), TCTI 2 4248: 3 (ŠS 5/-), ZA 25 S. 210 3: 5 (IS $1 /$-), TÉL 63: II 16 ([?] / [?]), CT 74217757 IV 1 (-/-), OrSP 47/49 500 Rs. 129 (-/-), TCS 1 147: 8 (-/-); KU zì: Rochester 231: 5 (ك̌ $46 /$ xi -), MVN 12 115: 3 (Š 46/ -), u. KU zì-gú-na: TIM 614 Rs. 4 (ŠS 6 / -).
} 
An dritter Stelle wäre anzuführen, daß zì-KA in Umma zì-gú-na, 'Mehl (der) gú-na(-Steuer)' ${ }^{14}$, zu entsprechen scheint ${ }^{15}$. Dafür spricht gleichfalls, daß zì-gú-na in Lagaš bis jetzt nicht nachgewiesen werden konnte und beide Termini zudem in ähnlichen Zusammenhängen vorkommen. Als Beispiel möchte ich den Lagaš-Text $C T$ 102014308 ( $\breve{S} 48$ / i-xii -) und die beiden Umma-Texte MVN 21 200 (AS $2 /$-) und STA 2 (AS 4 / -) anführen. Der erste Text ist eine Abrechnung von Mehl und Bier des Stadfürsten Urlama, die das Haus der Nindingir-Priesterin der Baba anbetrifft. MVN 21200 und STA 2 sind dagegen Abrechnungen über Dienerinnen als Arbeitskräfte, die auch für die Mehlerzeugung tätig waren. Die drei Texte erwähnen Dienerinnen, die für zì-KA / zì-gú-na eingesetzt waren:

1) CT 1020 14308: III 36-37:

1200 géme $\mathrm{u}_{4}-1-$ šè,

á zì-KA é-ta è-a nin-dingir-ra,
«(Entsprechende Arbeit von) 1200 Dienerinnen für einen Tag,

Arbeitskräfte der Nindingir-Priesterin, die zì-KA(-Gabe) aus dem Haus herausgebracht haben».

\footnotetext{
${ }^{14} \mathrm{Zu}$ gú-na $/$ gú-un = biltum als eine Art von Steuer s. $A H w$ I, S. 126 biltu $(m)$, «Tragen, Last; Talent; Ertrag; Abgabe» u. CAD B, S. 229 biltu «...4. tax (payable to the king), rent (payable to the lessor of a field or garden), 5. tribute (paid by subjected rulers)», s. ferner gú(-un-ma-da) bei P. MicHALOWSKI, «Foreign tribute to Sumer during the Ur III Period», ZA 68 (1978) S. 34 ff. u. P. STEINKELLER, «The Administrative and Economic Organization of the Ur III State: The Core and the Periphery», The Organization of Power: Aspects of Bureaucracy in the Ancient Near East, SAOC 46, Hrg. McG. GIBSON - R. D. BIGGS (Chicago 1987) S. $30 \mathrm{ff}$.

${ }^{15}$ zì-gú(-na) ist mir in folgenden Texten aus Umma bekannt: SAT 2 585: 2 (ॅ̌ 48 / x -), MVN 21 200: I 8 (AS 2 / -), JCS 31 S. 1342 Rs. 24 (AS 4 / -), MVN 10 219: I 4 (AS 4 / -), STA 2: I 10, 15, 18, II 1, 4 (AS 4/ -), MVN 10 204: 5, Rs. 7' (AS 5 / viii -), AAICAB 1/1 Ashm. 1924-651: 1, Rs. 4 (AS 8 / -), Nik 2328 Rs. 2 (AS 8 / -), Buffalo SNS 11/2 S. 1171 Rs. IV 4 (ŠS $1 /$ ii -), BCT 2 337: 1, Rs. 10

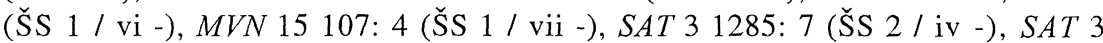
1430: 1 (ŠS 4 / -), TIM 6 14: 1, Rs. 4 (ŠS 5 / -), CST 708: 2 (ŠS 6 / vi -), Torino 2 474: 1 (ŠS 6/-), MVN 16 1374: 1 (ŠS 7 / iii -), MVN 15 356: 6 (ŠS 9 / iii -), SAT 3 1874: 1 (ŠS 9 / -), MVN 21412 Rs. VIII 4 (IS 1 / -), Michail 66: 1 (IS 2 ) xii 2), Michail 58: 1 (IS 2 / xii 6), SNATBM 531: 2 (IS 3 / i -), OrAnt 11 S. 274 3: 1, Rs. 24 (IS $3 /$ iii -), Nik 2 273: 3 (-/-), SET 270: I 1, «Edge» (-/-) u. SNATBM 536: II 8 (-/-).
} 
2) $M V N 21$ 200: I 7-8:

27490 lá 1 géme $u_{4}-1$-šè,

á-zì-gú-na-uru-ka,

3) STA 2: I 24-II $1^{16}$ :

1190,50 géme $u_{4}-1$-šè,

á-zì-gú-na-ka,
«(Entsprechende Arbeit von) 27489 Dienerinnen für einen Tag, Arbeitskräfte (für) Mehl (der) gú-na(Steuer) der Stadt».

Sollte dies stimmen, so ist davon auszugehen, daß Realien+KA, die nur in Lagaš bezeugt sind (amar-áb-KA ${ }^{17}$, dabin-KA ${ }^{18}$, še-KA ${ }^{19}$ und udu-KA ${ }^{20}$ ), ihre Entsprechungen in Umma in Realien+gú-na haben ${ }^{21}$. Im Falle von še-KA (Lagaš) für še-gú-na (Umma) ist jedoch zu beachten, daß der in Umma bezeugte Ausdruck še-gú-na

${ }^{16}$ Der Text führt Arbeitskräfte für Mehl der gú-na(-Steuer) in der Stadt Umma (Vs. I 9-10), Apisal (Vs. I 14-15, 18-19), in einem ungenannten Ort (Vs. I 24-II 1) u. Mušbiana (Vs. II 3-4) an. Als Beispiel sei nur Vs. I 24-II 1 erwähnt, da die Zahl der Arbeitskräfte in CT 1020 14308: III 36-37 sehr ähnlich ist.

${ }^{17} A A S$ Pl. LIX-LX CFC 110 = No. 186: IV 5, Rs. III 12 ( Š $9 /$-).

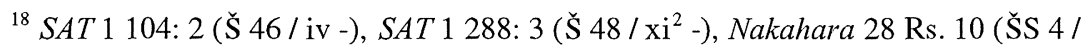
-), MVN 5253 Rs. 9 (- / xi 15), DAS 187: 12 (-/ $\left.\mathrm{xi}^{2} 13\right)$ u. RA 19 S. 41 L Rs. 5 (-/-).

${ }^{19}$ Hirose 343: 3 (ك̌ $35 /$-) u. AAS Pl. LXXII CFC $145=$ No. 199: 4 (ŠS $2 /$-).

${ }^{20}$ SAT 1 221: 4 (ŠS 8 / -).

${ }^{21}$ Während Zeugnisse für še-gú-na, Umma 46: 6 (Š 47 / ix -), SAT 31641 Rs. III 37, 40 (ŠS 6 / -) -hier (̌̌e-)gú-un-, SAT 3 1818: 5 (ŠS 7 / iii -) u. udu-gú-na, z.B. JCS 35 S. 208 8: 4 (ŠS 5 / iv -), BCT 2 19: 1, 4 (ŠS 8 / [ ]), in Umma nachweisbar sind, sind mir Belege für dabin-gú-na u. amar-áb-gú-na für Umma nicht bekannt. Die Existenz weiterer Realien+KA, z.B. síg-KA u. túg-KA, deren Entsprechungen síg-gú-na u. túg-gú-na in Umma belegt sind, konnte ich für Lagaš nicht nachweisen. Zu túg-KA in HLC 72 Pl. 81: III 3 (ك̌ $48 /$-), vgl. H. WAETZOLDT, $U N T$, S. 119, beachte Kollation von T. MAEDA, ASJ 2 (1980) S 209. Ferner beachte sipa-KA in dem Lagaš-Text $M V N 10162$ Rs. I 15' (ŠS 2 / -), gehört hierher? 
nur einmal in Lagaš belegt ist ${ }^{22}$. Diese Beobachtung zusammen mit der Tatsache, daß sich gú-na / gú-un ${ }^{23}$ in Lagaš nachweisen läßt, wobei ich zudem keinen Beleg der Variante KA für gú-na / gú-un kenne, halte ich eine Lesung zì-gun ${ }_{x}$ oder zì-gù für zì-KA für unwahrscheinlich. Vielmehr deutet das Ganze auf zì-KA als Gabe in Form von Mehl. Weitere Unterstützung für zì-KA als Gabe findet sich in folgenden Verbindungen:

1) $M V N 777: 1-2(\check{S} 40 /-)$ :

3.1.0.5 sìla še gur-lugal,

zì-KA-šè ak(-dè),
«965 1 Gerste (nach dem) königlichen (Maß gemessen),

(um) die zì-KA(-Gabe) (zu) machen» ${ }^{24}$.

2) Mehl, vor allem Gerstenmehl (dabin), wird für zì-KA 25 verwendet oder als zì-KA ${ }^{26}$ bezeichnet. In diesen Fällen kann zì-KA nicht nur Mehl oder eine Mehlsorte meinen.

${ }^{22}$ ASJ 3 S. 50 3: 3, Rs. 1 ( $\breve{S} 42$-AS 3 /-).

${ }^{23}$ gú-na ist in folgenden Termini belegt: gú-na, UDT 42: II 27, Rs. III 42 (AS $1 /$-), RTC 306 Rs. I 8 ([?] / [?]) u. AuOr 17-18 S. 228 No. 39 Rs. 3 (-/-); na gú-na, $M V N 11$ 134: 4 (AS 2 / ix -); níg-gú-na = unūtu(m), 'Geräte', s. $A H w$ III, S. 1422 unūtu(m), «Gerät(e), Utensilien; Mobiliar», u. D. I. OweN, «Widow's rights in Ur III Sumer», ZA 70 (1981) S. 182 Anm. zu II 1, z.B. níg-gú-na-giškin-ti, MVN 1769 Rs. 7 (- / iii 7) u. CT 74717775 Rs. 7 (- / iii 13); Feldname a-šà-gú-na, $A S J 17$ S. 216 Rs. I 7' ([ ] / [ ]); PN Ur-gú-na, MVN 17 55: II 15 (AS 4 / -), u. wohl PN auch Lú-gú-na, MVN 2 233: 5 (- / xi 6).

${ }^{24}$ Zur Konstruktion des Absolutiv (= Herstellungsmaterial) Terminativs (= Endprodukt) bei den Verben des Machens s. A. FALKEnsteIn, Grammatik der Sprache Gudeas von Lagaš. 2. Syntax, AnOr 29/2 (2. Ed. Roma 1978) S. 103 f. Da àr(-e)-dè mit Mehl zu erwarten ist, nehme ich an, daß es hier die Vorbereitung der Mehlgabe betont wird, vgl. TRU 295 Rs. 21 (Š 47 / xi 20): níg-mí-ússa-šè ak-dè, '(Vieh), um die Gabe (des) Schwiegersohn(s) zu machen'.

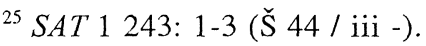

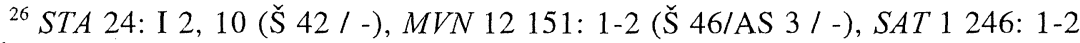
(Š 47 / xi -), MVN 17 144 = MVN 12 356: 1, 4 (AS 2 / ii -), TCTI 2 3980: 1-2 (AS 8 / -), TCTI 2 3946: 1 (ŠS $1 /$ ii -), TCTI 2 4242: 1-2 (ŠS 8 / xii -), ITT 2 2778: 1, Rs. 4-5, 7 (ŠS 9 / -), -), MVN 2 264: 1, 3, 5 (IS 1 / -), AuOr 17-18 S. 223 No. 22: 3-4 (-/-). MVN 2 188: 1, Rs. 3 (-/-) u. Priests S. 101 f. Appendix 4a: I 1 , Rs. V $1(-/-)$. 
3) Die Deutung von zì-KA als Gabe erklärt darüber hinaus den Umstand, daß die Empfänger von zì-KA (šu...ti / kišib) administrative Tätigkeiten ausüben: dub-sar-hé-dab ${ }_{5}{ }^{27}$, 'Schreiber (der) hédab $_{5}$-Leute', dub-sar-zì-da ${ }^{28}$, 'Schreiber des Mehls', engar nu-bànda$\mathrm{gu}_{4}$ é- ${ }^{\mathrm{d}}$ Šul-gi ${ }^{29}$, 'Pflugbauer (und) Domäne-Oberaufseher (des) Tempel(s) (von) Šulgi', nu-bànda-gu ${ }_{4}{ }^{30}$, 'Domäne-Oberaufseher', sagi- ${ }^{\mathrm{d}}$ Gù-dé-a ${ }^{31}$, 'Mundschenk des Gudea', sanga- ${ }^{\mathrm{d}}$ Ig-alim ${ }^{32}$, 'Sanga-Verwalter (des) Igalim', sanga- ${ }^{\mathrm{d}}$ Dumu-zi ${ }^{33}$, 'Sanga-Verwalter (des) Dumuzi', [sanga?]- ${ }^{\mathrm{d}}$ Nin-giš-zi-da ${ }^{34}$, '[Sanga-Verwalter'] des Ningišzida', ša ${ }_{13}$-dub-ba ${ }^{35}$, 'Archivist', ugula ${ }^{36}$, 'Aufseher', und nubànda ${ }^{37}$, 'Oberaufseher'.

Obgleich zì-KA ursprünglich eine Gabe in Form von Mehl bezeichnete, scheint es, daß es auch Gaben in Form anderer aus Getreide verarbeiteten Produkten, wie etwa Brot, bezeichnete:

TCTI 22609 (-/-):

158.0.0 ninda gur, $\ll 474001$ Brot, ninda àga-ús, Brot (für ${ }^{2}$ ) die Leibwächter;

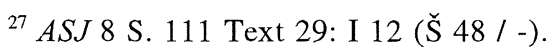

${ }^{28}$ Amherst 59: II 3, Rs. II 1 = BCT 2 286: II 3, Rs. II 1 (AS $1 /$-), TUT 118 Rs III 17-18 (AS 1/-), CT 104418962 Rs. 1-3 (-/-) u. MVN 6 285: 4-5, 8 (-/-).

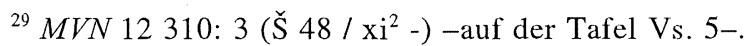

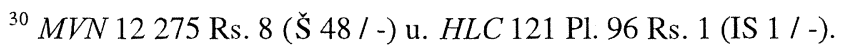

${ }^{31}$ MVN 12323 Hülle: 3, Siegel (AS $1 /$ ii -).

${ }^{32}$ TCTI 2 4297: 4 (ŠS 1 / xii -).

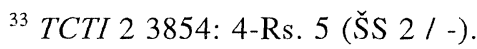

${ }^{34}$ TCTI1 687 Rs. 7-8 (ŠS $3 /$-).

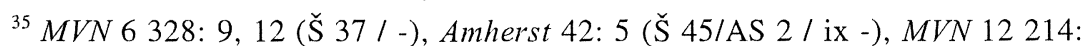

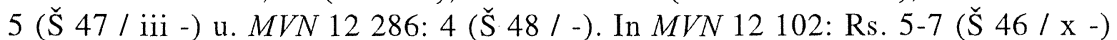
empfängt Ur-Ma-ma die Gerste in Namen von ${ }^{\mathrm{N}} \mathrm{Nanše-kam,} \mathrm{dem} \mathrm{Archivist} \mathrm{(} \check{\mathrm{s}} \mathrm{a}_{13^{-}}$ dub-ba)

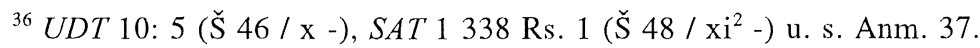

${ }^{37}$ MVN 12319 Rs. 6 (AS 1 / -). In TCTI 2668 Rs. 5-6 (ŠS 9 / xi -) erhält Lú- ${ }^{d} B a-$ $\mathrm{ba}_{6}$ die Gerste in Namen der Aufseher und Oberaufseher (mu ugula nu-bànda-ne- Šè).
} 


$$
\begin{array}{ll}
\text { šà Gír-su }{ }^{\text {ki }}, & \text { in Girsu; } \\
\text { zì-KA-ta, } & \text { aus der zì-KA(-Gabe) (abgeliefert)». }
\end{array}
$$

Um die Art dieser Gabe zu bestimmen, möchte ich drei Textgruppen in Betracht ziehen:

1) Priests S. 101 f. Appendix $4{ }^{38}$ führt Mehlangaben vor Berufen an, wonach die gesamte Summe (Rs. IV 1) 740601 beträgt. Der Summe folgt der Vermerk (Rs. V 1-4) zì-KA uru-a gar-ra, šà Gú-ab-ba ${ }^{\mathrm{ki}}$, ù é- ${ }^{\mathrm{d}}$ Inanna, 18/18 iku 0.2.0 lugal-ta, «zì-KA(-Gabe), die 'in die Stadt gesetzt worden ist', in Guabba und in dem Tempel Inannas; (Abgabeverhältnis:) je 1 bùr (ca. 6,48 ha) mit 1201 Mehl (nach dem) königlichen (Maß gemessen)». Hier stellt sich die Frage, ob diese Personen das Mehl bekamen oder sie es geben sollten ${ }^{39}$. Angesichts der Feldflächenangabe sollten diese Personen das Mehl wohl geben, da sich die Feldfläche sehr wahrscheinlich auf Felder bezieht, die sie bebauten (Pachtfelder? ${ }^{40}$. Diese erhielten sie von der Administration bzw. den Tempeln in Guabba und dem Tempel Inannas. Dabei hing ihre Gabe in Form von Mehl (hier wohl eine Art von Steuer) von der Fläche der Felder $a b^{41}$.

\footnotetext{
${ }^{38}$ Der Text ist von K. Maekawa, Priests, S. $89 \mathrm{ff}$. behandelt worden.

${ }^{39} \mathrm{Vgl}$. K. MAEKawA, Priests, S. 89 X. 4 «..flour (ZÍD.KA) which was given(?) to the people...».

${ }^{40}$ Hier ist $A S J 9$ S. 332 f. Text 8 (ŠS $8 /$-) zu beachten, der zì-KA im Rahmen von Pachtfelder anführt. Der Text erwähnt, wenn ich ihn richtig verstehe, Gerste, die engar, 'Pflugbauer', u. šà-gu ${ }_{4}$, 'Pflugführer', im Bereich von 4 Pachtfeldern auf dem Felde a-šà ù-a-du $10^{-}$ga (Rs. 15) erhalten. Die Gerste wird in drei Gruppen gegliedert, die nach der gesamten Summe (Rs. 10-12) folgende ist: $6810 \mathrm{l}$

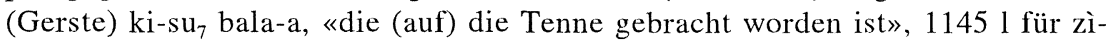
KA(-Gabe) und $158 \mathrm{l}$ (für) die Löhne der Mietlinge. Hier scheint mir, daß zì-KA sowie in den sogenannten «seed-and-fodder texts» eine Art von Gabe wegen bestimmter Arbeiten (s. unten) ist.

${ }^{41}$ Der Ausdruck zì-KA uru-a gar-ra ist mir sonst nur in ITT 5 6811: 2-5 (IS 2 / -) bekannt, in dem auch eine Beziehung zur Feldfläche besteht: pisan-dub-ba, zì-KA urua gar-ra, 18/18 iku 1.1.0.-ta, ù zì-KA-lú-še-ba, ì-gál, «Tafelbehälnis: zì-KA(-Gabe), die 'in die Stadt gesetzt worden ist'; (Abgabeverhältnis:) je 1 bùr (ca. 6,48 ha) mit 3601

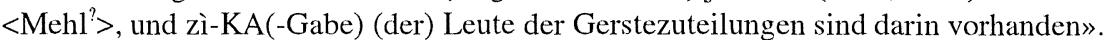
Eine Verkürzte Form dieses Ausdrucks ist höchstwahrscheinlich zu sehen in zì-KA-

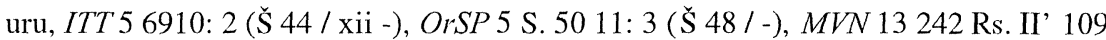
([ ] / [ ]), u. dabin-KA-uru, DAS 187: 12 (- / $\left.\mathrm{xi}^{2} 13\right)$, vgl. oben Anm. 11. uru-a gar-ra,
} 
Ein dem Priests S. 101 f. Appendix 4 ähnlicher Text ist $M V N 2$ 188: 1-Rs. 4 (-/-):

[ ] dabin gur-lugal,

$[$ šit $] a_{x}{ }^{2}-a b-b a$,

[ ]x àga-ús-šita ${ }_{x}-a b-b a$,

[ ] má-DU 0.1.3-ta,

4 ì-du 8 0.1.3-ta,

2 ga-íl 0.1.3-ta,

0.1.[3] muš-lah

0.1 .3 ad-KID,

0.1 .3 sagi,

[ ]+5 sìla ugula-nar,

[ ]+5 sìla LÚ.[x],

0.1 .3 ì-[rá $\left.{ }^{2}-\mathrm{ra}^{2}\right]$,

[0.1.]3 A-x-x //

[]LÚ-[ ],

[ ]x.AB.[?],

zì-KA zi-zi-dam,

šà Gú-ab-ba ${ }^{k i}$

«[ ]+300 1 Gerstenmehl (nach dem) königlichen (Maß gemessen)

$\left(\right.$ des $\left.^{3}\right)$ Šitaabba' - Priester(s) ${ }^{42}$

[ ] (Gerstenmehl) (des $\left.{ }^{2}\right)$ Leibwächter(s) des Šitaabba-Priesters,

[ ] Schiff-... je mit 901 (Gerstenmehl),

4 Pförtner je mit 901 (Gerstenmehl),

2 Käseträger je mit 901 (Gerstenmehl),

901 (Gerstenmehl) (des $\left.{ }^{2}\right)$ Schlangentreiber(s),

901 (Gerstenmehl) (des ${ }^{\text {) }}$ Rohrflechter(s),

901 (Gertenmehl) (des? $\left.{ }^{2}\right)$ Mundschenk(en),

[ ]+5 1 (des $\left.{ }^{2}\right)$ Aufseher(s) (der) Sänger,

[ ]+5 1 (des') LÚ.[x],

901 (Gerstenmehl) (des? $)$ Ölmischer(s? $)$,

901 (Gerstenmehl) (des? $\left.{ }^{?}\right)$ A-x-x,

[ ]

[ ]

(diese) zì-KA(-Gabe) ist abzuliefern;

in Guabba».

Obgleich keine Feldfläche angegeben wird, läßt der Parallelismus zu Priests S. $101 \mathrm{f}$. Appendix 4 annehmen, daß das aufgeführte Personal der Verwaltung auch Mehl abgeben sollte.

«in die Stadt setzen», meint wohl, daß die Gabe, in diesem Falle das Mehl, schon bezahlt worden ist. Zu uru in anderen Verwaltungsausdrücke s. z.B. uru-ta nu-è bei K. MAEKAWA, «New Texts on the Collective Labor Service of the Erín-People of Ur III Girsu», ASJ 10 (1988) S. 59 ff.

${ }^{42}$ Aufgrund des Textaufbaus ist ein Beruf in der zweiten Zeile zu erwarten. Da nur Platz für ein Zeichen vor ab-ba besteht, ist wahrscheinlich šita-ab-ba zu ergänzen. Zu šita-ab-ba als eine Art von Priester s. R. A. HenshAw, Female and Male. The Cultic Personnel. The Bible and the Rest of the Ancient Near East (Allison Park, Pennsylvania 1994) S. 34 1.9.6. Der Beruf ist mir während der UrIII Zeit nur für die Provinz Lagaš bekannt: CT 52518346 Rs. VI 16 (Š 46 / -), MVN 12 3: 3 (Š $46 /$-), TCTI 1871 Rs. VII 17' (ŠS $3 /$-), ASJ 13 S. 235 Text 76 Rs. II' 14' ([ ]/ [ ]), TCTI 1 878: III 10 ([ ] / [ ]), ASJ 20 S. 105 Text 5 Rs. III 12 (-/-), Priests S. 101 Appendix 4a: III 19, V 2 (-/-) u. Zinbun 21 Pl. X Text 43 Rs. I 9' (-/-). 
2) Eine Beziehung zwischen zì-KA und Feldern läßt sich ebenso nachweisen in einer Gruppe von Texten, die K. Maekawa als «seedand-fodder texts» bezeichnet hat. Die Texte erwähnen im Rahmen der Säarbeiten zwischen 1201 und 24001 Gerste (für) si ù zì-KA(éren-na) ${ }^{43}$, 'si(-Gebäcke?) ${ }^{44}$ und zì-KA(-Gabe) (der Arbeitstruppen)'. Da diese Texte eine Berechnung von Getreide darstellen, die bei den Säarbeiten gebraucht wird, scheint mir kaum möglich, daß zì-KA in diesem Kontext eine Steuer meint, vielmehr dagegen eine Gabe für von Arbeitstruppen geleisteter Arbeit zu sein.

Hierbei ist zu erwähnen, daß zì-KA vereinzelt Verwendung fand für die Gerstezuteilungen der Arbeiter:

MVN 20 119: III' 6'-9' ([ ] /[ ]):

14.1.0.0 še zì-KA <gur><a- $\quad$ «260 1 Gerste (für) zì-KA(-Gabe) aus dem šà->GÍR-gunû.GÍR-gunû-ta, Feld a-šà-GÍR-gunû.GÍR-gunûu ${ }^{45}$,

${ }^{43}$ ASJ 3 S. 554 Rs. I 9', II 10' (Š 33 / -), ASJ 17 S. 209 Text 102 Rs. I 13, II 8 (Š 33 / -), CT 71515324 Rs. III 14, IV 12 (Š 33 / -), CT 917 12917: II 20, Rs. IV 11 ( $\breve{S} 33 /$-) u. STA 28 Rs. III 5, IV 5 ( $\breve{S} 33 /$-). Zu den sogenannten «seed-and-fodder texts» s. K. MAEKAWA, «Cereal Cultivation in the Ur III Period», BSA 1 (1984) S. 75 ff., u. IDEM, «The Girsu 'Seed-and-Fodder Texts' of Šulgi 41», Zinbun 24 (1989) S. 135 ff. K. MAEKAwA hat Texte aus dieser Gruppe in verschiedenen Aufsätzen in $A S J$ veröffentlicht: $A S J 3$ (1981) S. 37 ff., $A S J 8$ (1986) S. 85 ff., $A S J 9$ (1987) S. 89 ff., ASJ 11 (1989) S. 114 ff., ASJ 15 (1993) S. 107 ff., u. $A S J 17$ (1995) S. 175 ff. Dabei ist zu beachten, daß Gerste (für) si ù zì-KA(-éren-na) bis jetzt nur in jenen Texten vorkommt, die Š 33 datieren. Andererseits sind diese mit Ausnahme von CT 917 12917, wo kein Vermerk steht, die einzigen, die den Vermerk enthalten níg-ŠID-ak še-numun mur-gu ${ }_{4}$ álú-huun-gá ù še-amar, «Abrechnung (von) Sägetreide, (des) Ochsenfutter(s), (der) Löhne (der) Mietlinge und (der) Kälber». Nur $M V N 1190$ Rs. 34-35, der Š 33 datiert und jenen Vermerk enthält, führt Gerste (für) si ù ŠÈ.KA(-éren-na) nicht an. In Verbindung mit Säarbeiten ist auch $A S J 9 \mathrm{~S}$. $332 \mathrm{f}$. Text 8 (ŠS $8 /$-) zu sehen aufgrund des Vermerks še-šuku-ra engar ša-gu ${ }_{4}$, 'Verpflegungsgerste (der) Pflugbauer (und) Pflugführer'.

${ }^{44}$ Die Deutung von si als eine Art von Gebackenem gründet sich auf UDT 54: 13, Rs. 16 ( $\breve{S} 42 /-$ ), in dem Gerste für si-gal nach zì-KA angeführt wird. Zu sigal als eine Art von Gebackenem s. M. CIVIL, «A Hymn to the Beer Goddess and a Drinking Song», in Studies Presented to A. Leo Oppenheim, Hrg. R. D. BIGgS u. J. A. Brinkman (Chicago 1964) S. 77 Anm. zu Z. 13-20.

${ }^{45}$ Zum Feld a-šà-GÍR-gunû.GÍR-gunû s. z.B. G. Pettinato, Untersuchungen zur neusumerischen Landwirtschaft. I. Die Felder. 1 (Napoli 1967) S. 250 f. 
mu géme-uš-bar-e še-ba-šè šu

ba-ti- ${ }^{?} \mathrm{a}^{?}<-$ šè $>$,

$\mathrm{Ku}-\mathrm{li}$ dumu Ki-ág-gu ${ }_{10}$, šu ba-ti, weil die Weberinnen für die Gerstezuteilungen in Empfang genommen haben, hat Kuli, der Sohn (des) Kiaggu, in Empfang genommen».

3) HLC 32 Pl. 67 (AS 1 / -), TCTI 23723 (AS 2 / -) und TUT 111 (AS $2 /-)^{46}$. TUT 111 ist eine Abrechnung über ausgeliehene Gerste (níg-ŠID-ak-še-ur $r_{5}$-ra), u.a. an das Personal der Haupttempel (Vs. IRs. IX 1) -hier entspricht TCTI 23723 TUT 111 Rs. VIII 3-11- und an verschiedene Arbeitsgruppen (Rs. IX 10-XII 22). Der letztgenannte Abrechnungsteil findet eine ähnliche Parallele in der ein Jahr früher datierten Passage HLC 32 Pl. 67: I-Rs. III 15.

TUT 111 führt die vorhandene Gerste an, von der der Teil, der abgerechnet wird, als zì-KA und das Übrig gebliebene als lá-NI, 'Rest' ${ }^{47}$, bezeichnet wird. Im Folgenden möchte ich einige Beispiele anführen:

\begin{tabular}{|c|c|c|c|}
\hline Rs. X 6-9 ${ }^{48}$ & Vorhandenes & zì-KA & 1á-NI \\
\hline nu-bànda Gù-dé-a & 156461 & 91501 & 64961 \\
\hline
\end{tabular}

Wie die Tabelle zeigt, verfügt der Oberaufseher Gudea über 156461 (=9150 + 64961$)$, von denen er für zì-KA 91501 verwendet, so daß 64961 übrig bleiben.

Bei den Hauptheiligtümern wird das Personal, das die Gerste gebraucht, erwähnt. Sie werden in ab-ba-ab-ba, die 'ältesten' ${ }^{49}$,

${ }^{46} \mathrm{Zu}$ den Texten HLC 32 Pl. 67 u. TUT 111 s. K. MAEKAwA, RA 70 (1976) S. $11,13 \mathrm{ff}$.

${ }^{47}$ Zum problematischen Terminus lá-NI s. Literatur bei M. SUCH-GuTIÉRREZ, «Eine ungültig gemachte Abrechnung aus Umma», ASJ 21 (1999) Anm. 2 (im Druck).

${ }^{48}$ Diese Passage entspricht $H L C 32$ Pl. 67: I 16-18.

${ }^{49} \mathrm{Zu}$ ab-ba-ab-ba als 'altästen' im Sinne höchster administrativer Stellen im Tempels s. R. de MAaiJer u. B. Jagersma, Rezension zu PSD A/1-2, AfO 44-45 (1997-1998) S. 287 ab-ba A u. K. MaeKawa, Priests, S. 80 f., vgl. IdeM, «Ur III Girsu Records of Labor Forces in the British Museum (1)», ASJ 20 (1998) S. 79 II.4. Zu abba s. ferner G. J. SELZ, Altsumerische Verwaltungstexte aus Lagaš. Teil 2. Altsumeris- 
engar nu-bànda-gu ${ }_{4}$, 'Pflugbauern (und) Domäne-Oberaufseher' und éren, 'Arbeitstruppen', gegliedert:

\begin{tabular}{|l|r|r|r|r|}
\hline Vs. I 1-30 & \multirow{2}{*}{ Vorhandenes } & \multicolumn{2}{|c|}{ zì-KA } & \multicolumn{1}{c|}{ lá-NI } \\
\hline & & é-$^{d}$ Nin-gír-su & é-šabra & \\
\hline ab-ba-ab-ba & 180001 & 116201 & 60001 & $4401^{50}$ \\
\hline engar nu-bànda-gu & 1098001 & 289201 & 131801 & 677001 \\
\hline éren & 520351 & 186901 & 120801 & $212051^{51}$ \\
\hline Summe & 1798351 & 592301 & 312601 & $893451^{52}$ \\
\hline
\end{tabular}

Die 3 Gruppen verfügen über bestimmte Beträge von Gerste, von denen sie insgesamt nur $904901(=59230+312601)^{53}$ als zì-KA benötigten; das Übrig gebliebene (89345 1) wird als lá-NI bezeichnet.

Wenn die Zahl der benötigten Gerste höher als die vorhandene ist, wird der Überschuß als diri...sum-mu, 'Überschuß zu geben' ${ }^{54}$, bezeichnet:

\begin{tabular}{|l|c|c|c|}
\hline Rs. X 14-18 & Vorhandenes & zì-KA & diri...sum-mu \\
\hline nu-bànda Ur-ša 6 -ga gu-za-lá & 112551 & 170701 & 58151 \\
\hline
\end{tabular}

che Wirtschaftsurkunden aus Amerikanischen Sammlungen. 2, FAOS 15/2-2 (Stuttgart 1993) S. 546 Anm. zu 3:10, u. PSD A/2 S. 129 ff.

${ }^{50}$ Die Zahl sollte 3801 sein: $18000-176201(=11620+60001)=3801$.

${ }^{51}$ Die Zahl sollte 212651 sein: $52035-30770 \mathrm{l}(=18690+12080 \mathrm{l})=21265 \mathrm{l}$.

${ }^{52}$ Beachte, daß diese Summe nicht der im Text stehenden Anzahl (85015 1, Vs. I 29) entspricht.

${ }^{53}$ Beachte, daß die Zahl nicht zu jener paßt, die im Text steht (90430 1, Vs. I 20).

${ }^{54} \mathrm{Zu}$ diri als 'Überschuß' s. R. K. ENGLUND, Organisation und Verwaltung der Ur III - Fischerei, BBVO 10 (Berlin 1990) S. 48 ff. 
Der Oberaufseher Uršaga hat 58151 mehr benötigt, als zur Verfügung stand $(11255 \mathrm{l})^{55}$, so daß er später diese zu der zìKA(-Gabe) gehörigen Gerste geben wird (diri...sum-mu). Dies scheint dafür zu sprechen, daß die in TUT 111 genannten Personengruppen zì-KA geben sollten. Ein weiterer Hinweis ist, daß der ein Jahr früher datierende Text HLC $32 \mathrm{Pl} .67$ Rand (AS 1 / -) den Vermerk Ur- ${ }^{\mathrm{d}}$ Nun-gal ì-dab ${ }_{5}$, «Urnungal hat gepackt (genommen)», im Anschluß an zì-KA (und lá-NI) enthält.

\section{Zİ-KA IN DEM ALTAKKADISCHEN TEXT FAOS 19 S. 44 FF. AD $5(-/-)$}

In dem oberen Abschnitt habe ich zì-KA in den Quellen aus der Ur-III Zeit, in denen der Terminus eine Art von Gabe bezeichnet, behandelt. Jedoch läßt sich zì-KA zum ersten Mal in einem altakkadischen Brief aus Adab, FAOS 19 S. 44 Ad 5: 2 (-/-), wenn die Transliteration stimmt, nachweisen. Im Gegensatz zu der Ur-III Zeit bezeichnet zì-KA in dem Brief eine Mehlsorte ${ }^{56}$. Sollte diese Deutung von zì-KA als eine Art von Mehl durch neue Texteditionen weiter bestätigt werden, wäre eine Deutungsentwicklung -von Mehlsorte (altakkadische Zeit) zu Gabe (Ur-III Zeit)- anzunehmen, es sei denn, daß zwei verschiedene Lesungen für das Zeichen KA liegen.

\section{SCHLUßFOLGERUNG}

In der Ur-III Zeit bezeichnete zì-KA ursprünglich eine Gabe in Form von Mehl, die später auch in Form anderer aus Getreide verarbeiteten Produkten, wie z.B. Brot, abgegeben werden konnte. In Lagaš entsprach wohl zì-KA dem für Umma belegten zì-gú-na, 'Mehl (der) gú-na(-Steuer)'. Während eine Deutung als 'zì-KA(-

\footnotetext{
${ }^{55}$ Ein Jahr früher, HLC 32 Pl. 67: II 2-3, werden die 112551 als zì-KA bezeichnet, vgl. K. MaEKawA, RA 70 (1976) S. 15 Anm. 6.

${ }^{56}$ Dies ergibt sich aus dem Zusammenhang, in dem zì-KA neben anderen Mehlsorten (zì-ba-ba, zì-za, zì-ìmgaga usw.) angeführt wird.
} 
Sef 63:2 (2003) ÜBERLEGUNGEN ZU DER SUMERISCHEN ZEICHEN-GRUPPE ŠÈ.KA 407

Steuer)' zwar für einige Zusammenhänge gut passen könnte, ist eine allgemeine Übersetzung mit 'zì-KA(-Gabe)' zu bevorzugen, da zì$\mathrm{KA}$ in anderen Passagen, wie in den sogenannten «seed-and-fodder texts», anscheinend der Zahlung für eine geleistete Arbeit diente. Dennoch scheint zì-KA in allen Fällen, in denen der Zweck bestimmt werden kann, in Verbindung mit Feldern gestanden zu haben. Diese Deutung als Gabe steht im Gegensatz zu dem ersten Beleg für zì-KA, nämlich dem altakkadichen Brief FAOS 19 S. 44 Ad 5: 2 (-/-), in dem der Terminus eine Mehlsorte bezeichnet. Dies läßt eine Deutungsentwicklung für zì-KA vermuten, es sei denn, daß KA von zì-KA in dem altakkadischen Brief und in den Ur-III-zeitlichen Texten aus Lagaš unterschiedlich zu lesen ist.

\section{APPENDIX: UR-III-ZEITLICHE BELEGE FÜR ŠÈ.KA IN ZUSAM- MENHANG MIT DER ABGABE VON NATURALIEN}

HLC 61 Pl. 1 Rs. III 18 (Š 9 / ii-xi -), ASJ 3 S. 554 Rs. I 9', II 10' (ک̌ 33 / -), ASJ 17 S. 209 Text 102 Rs. I 13, II 8 (Š $33 /$-), CT 71515324 Rs. III 14, IV 12 (ك̌ 33 / -), CT 917 12917: II 20, Rs. IV 11 (ك̌ 33 / -),

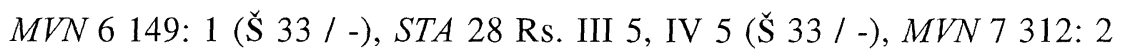

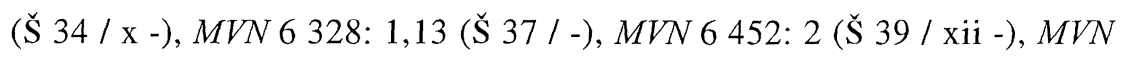

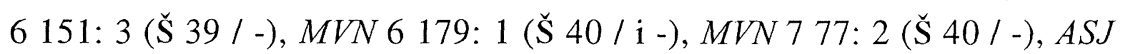

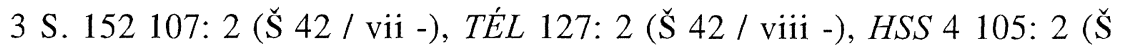

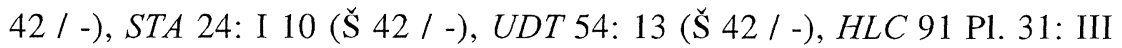

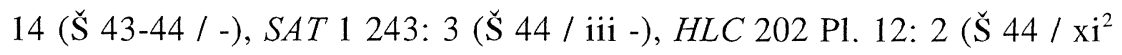

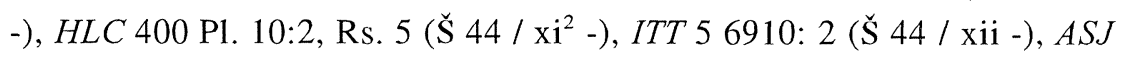
3 S. 159 125: 3 (ك̌ 44 / -), CT 519 12912: III 14, V 13, Rs. VII 31 (ك̌ 44

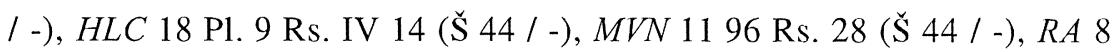
S. 87 AO 3636 Rs. 1 (Š 45 / ii -), ASJ 3 S. 168 154: 2 (ك̌ 45/AS 2/ viii -),

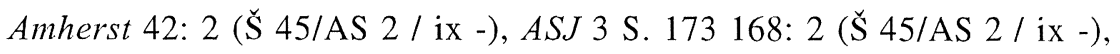

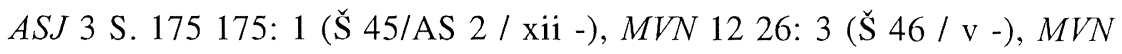



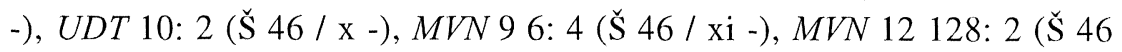

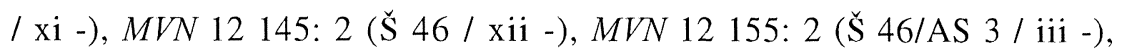




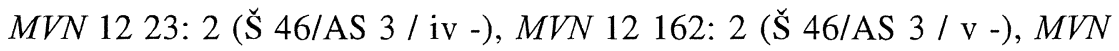

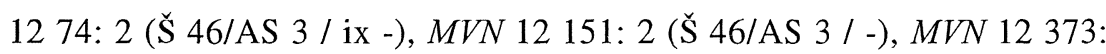

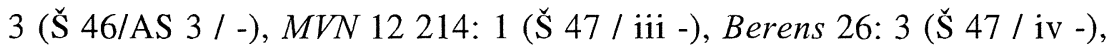

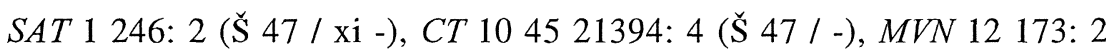

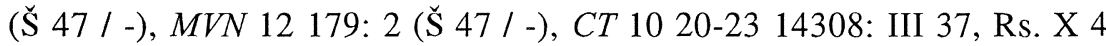

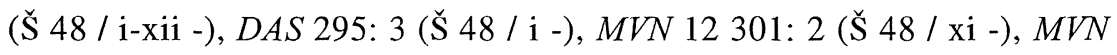
12 308: 2 ( $\breve{\mathrm{S}} 48 / \mathrm{xi}^{2}$-), MVN 12 310: 2 ( $\breve{\mathrm{S}} 48 / \mathrm{xi}^{2}$-) -in der Hülle Vs. 1-,

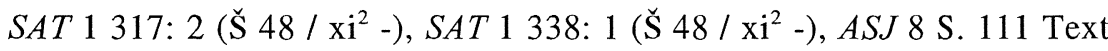

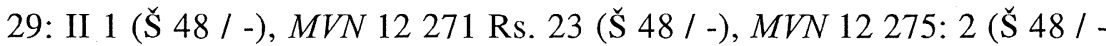

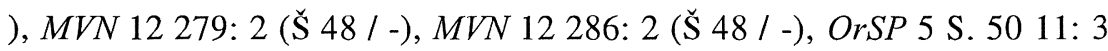
(Š 48 / -), MVN 12323 Tafel: 3 (AS $1 /$ ii -), Amherst 59 Rs. II $1=B C T 2$ 286 Rs. II 1 (AS 1 / -), HLC 32 Pl. 67: I 2, 6, 9, 12 passim (AS 1 / -), MVN 1184 Rs. 1 (AS 1/ -), MVN 12 315: 3 (AS 1/ -), MVN 12316 Rs. 10 (AS 1/-), MVN 12 319: 2 (AS 1/ -), TUT 118: II 9, Rs. IV 6 (AS $1 /$ -), MVN 17144 = MVN 12 356: 4 (AS 2 / ii -), TUT 182: 2 (AS 2 / ix -), TCTI 23723 Rs. 9 (AS 2 / -), TUT 111: I 21, II 31, III 26, IV 21 passim (AS 2 / -), MVN 956 «case»: 2 (AS 3 / iii -), RA 10 S. 65 20: 2 (AS 3 / xi -), MVN 12 391: 4 (AS 4 / -), TCTI 2 4134: 2 (AS 5 / -), TCTI 2739 Rs. 5 (AS 5/IS 4 / iv -), TCTI 24066 (AS 7 / xii -), Amherst 96: 1 = Orient 16 152: 1 (AS 8/xii -), Amherst 104: 2 (AS 8 / -), TCTI 2 3980: 2 (AS 8 / -), TCTI 2 3925: 2 (AS $9 / \mathrm{xi}^{2}$-), TCTI 2 4317: 1 (AS $9 / \mathrm{xi}^{2}$-), TCTI 2 3946: 1 (ŠS 1 / ii -), TCTI 2 2558: 3 (ŠS 1 / vii -) -in der Hülle Vs. 2-, TCTI 2

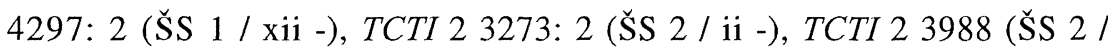
xi/xii -), TCTI 2 3854: 2 (ŠS 2 / -), HLC 12 Pl. 44: 2 (ŠS 3 / v -), TCTI 2

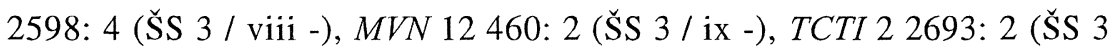
/ xi -), MVN 17 109: 2 (ك̌S 3 / -), TCTI 1 654: 2 (ŠS 3 / -), TCTI 1 687: 5 (ŠS 3 / -), TCTI 2 3275: 4 (ŠS 3 / -), TCTI 2 3681: 2 (ŠS 3 / -), TÉL 159:

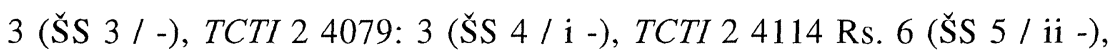

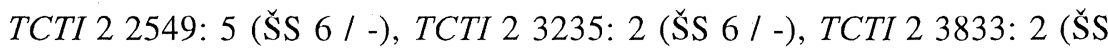
6/ -), MVN 5 189: 2 (ŠS 7 / iii -), TCTI 2 3639: 3 (ŠS 7 / -), TCTI 2 4101:

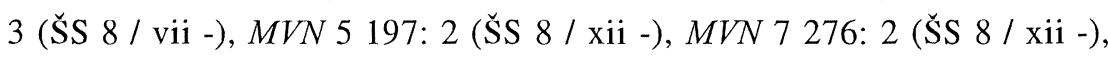
TCTI 2 4242: 2 (ŠS 8 / xii -), ASJ 9 S. 332 f. Text 8: 4, 9, 13, 18, Rs. 2, 6, 11 (ŠS 8/ -), TCTI 2 3579: 1 (ŠS 9 / ii -), TCTI 2 3779: 2 (ŠS 9 / ii -), TCTI 1 635: 3 (ŠS 9 / iii -), MVN 13 223: 2 (ŠS 9 / vii -), MVN 12 497: 2 
Sef 63:2 (2003) ÜBERLEGUNGEN ZU DER SUMERISCHEN ZEICHEN-GRUPPE ŠÈ.KA 409 (ŠS 9 / viii -), TCTI 2 3247: 2 (ŠS 9 / viii -), TCTI 2 2668: 2 (ŠS 9 / xi -), MVN 5212 Rs. 2 (ŠS 9 / xii -), TCTI 1947 (ŠS 9 / xii -), TCTI 2 3719: 2 (ŠS 9 / xii -), TCTI 2 3745: 2 (ŠS 9 / xii -), ITT 22778 Rs. 5, 7 (ŠS 9 / -), MVN 5 213: 2 (ŠS 9 / -), MVN 5 214: 2 (ŠS 9 / -), MVN 12 492: 2 (S̆S 9 / -), TCTI 2 2580: 3 (ŠS 9 / -), TCTI 2 3923: 3 (S̆S 9 / -), TCTI 2 4227: 3

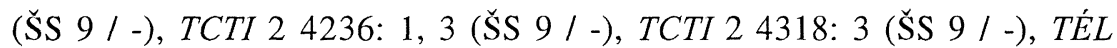
178: 2 (ŠS 9 / -), HLC 121 P1. 96: 1 (IS 1 / -), ITT 5 6786: 1 (IS 1 / -), MVN 2 264: 5 (IS 1/ -), TCTI 2 2667: 2 (IS $1 /$-), Orient 16 162: 3 (IS 1 / -), MVN 5 225: 5 (IS 2/ v -), TCTI 2 3635: 2 (IS 2 / xi -), TCTI 2 3177: 2 (IS 2 / xii -), ITT 5 6811: 2, 4 (IS 2 / -), TCTI 1 940: 4/5 (IS $2 /$-), TCTI 2 3232: 2 (IS 3 / xii -), MVN 19 82: 2 ([ ] / vii -), ITT 5 6903: 2, 4 ([ ] / xi -), MVN 5268 Rs. 5 (-/- 1), ASJ 2 S. 15 39: 3 ([ ] / [ ]), MVN 6106 Rs. 5 ([ ] / [ ]), $M V N 13242$ Rs. I' 71, 85, II' 109 ([ ] / [ ]), $M V N 17$ 60: III 14 ([ ] / [ ]), $M V N 17$ 68: II 11' ([ ] / [ ]), MVN 20 119: III' 6' ([ ] /[ ]), TUT 184: 3 ([ ] / [ ]), AuOr 17-18 S. 223 No. 22: 4 (-/-), CT 740 18430: 3 (-/-), CT 1044 18962: 3, Rs. 1 (-/-), MVN 2188 Rs. 3 (-/-), MVN 6 285: 8 (-/-), MVN 7 557: 2 (-/-), Priests S. 102 Appendix 4a Rs. V 1 (-/-), TCS 145 Rs. 1 (-/-), TCTI 2 2609: 4 (-/-) u. ZVO 4 2: 2 (-/-). 


\section{RESUMEN}

El presente artículo trata de las diferentes lecturas y significados del grupo de signos ŠÈ.KA. Entre dichas lecturas se encuentra la de Zì.KA o zì-KA/ka, que ha sido tradicionalmente interpretada bien como un tipo de harina, o bien como la acción que conduce a su fabricación. Sin embargo, una revisión de los textos de la III Dinatía de Ur (c. 2117-2008 a.C.) en los que aparece el término Zì.KA o zì-KA/ka demuestra que, en realidad, con él se hace referencia a un tipo de regalo.

Palabras Clave: Textos cuneiformes sumerios, III Dinastía de Ur, Lagaš, harina, regalo.

\section{SUMMARY}

This paper deals with the different readings and meanings of the signs ŠÈ.KA. One of these readings is Zì.KA or zì-KA/ka, which has been traditionally interpreted as a kind of flour or as the process of milling. However, a revision of the texts from the Third Dynasty of Ur (c. 2117-2008 B.C.), in which the group of signs Zİ.KA o zì$\mathrm{KA} / \mathrm{ka}$ appears, shows that this term is actually a kind of gift.

KEYWORDS: Sumerian cuneiform texts, Third Dynasty of Ur, Lagaš, flour, gift. 